\title{
How Intelligence Interviewees Mentally Identify Relevant Information
}

In principle acceptance by Peer Community in Registered Reports

https://doi.org/10.17605/OSF.IO/82QTN

\section{Author Note}

David A. Neequaye, Department of Psychology, University of Gothenburg, Gothenburg, Sweden.

Alexandra Lorson, School of Philosophy, Psychology and Language Sciences, University of Edinburgh, Edinburgh, United Kingdom

Correspondence to: David A. Neequaye, Department of Psychology, University of Gothenburg. Box 500, 40530 Gothenburg, Sweden; Email: david.neequaye@psy.gu.se

Data Availability Statement: All data supporting the findings in this research will be publicly available on the open science framework repository (osf.io).

Contributor roles:

- David A. Neequaye: Conceptualization, Data curation, Investigation, Methodology, Project administration, Writing - original draft, Writing - review \& editing

- Alexandra Lorson: Conceptualization, Data curation, Methodology, Formal Analysis, Writing - review \& editing

There are no conflicts of interest to declare.

The appendices appear after the reference list 
MENTALLY DESIGNATING INFORMATION ITEMS IN INTERVIEWS

\section{Abstract}

This research explores how intelligence interviewees mentally identify the relevant information at their disposal, which they may or may not disclose. We theorize that interviewees mentally identify applicable information items by estimating the interviewer's objectives based on how they frame any attempt to solicit information. Interviewees then mentally organize the information they possess into item designations that pragmatically correspond to the perceived interviewer-objective. The more an attempt specifies the interviewer's objective, the more the interviewee will mentally designate information items that pragmatically correspond with the perceived objective. We propose two studies to examine the theory. The findings would optimize further theorizing about how pragmatic considerations influence intelligence interview dynamics to possibly affect several downstream effects.

Keywords: disclosure; intelligence gathering; intelligence interviewing; pragmatic inference; relevance theory 
MENTALLY DESIGNATING INFORMATION ITEMS IN INTERVIEWS

\section{How Intelligence Interviewees Mentally Identify Relevant Information}

In human intelligence interviews, interviewers solicit information from interviewees for national or international security purposes (e.g, Brandon, 2014). This research examines how such interviewees mentally identify or designate applicable items of information in interviews ${ }^{1}$.

The thesis of this article is that during an interview, interviewees mentally designate applicable items of information. Designations here refer to an interviewee's mental representation of a coherent unit of information. That is, information that, if the interviewee desires, they can make the interviewer aware of, or ideally communicate clearly and logically ${ }^{2}$. Interviewees may consider such designations to be different or separate from other similarly designated information items. However, different information items need not be mutually exclusive. Interviewees can view information items as separate but related units when events induce links between the items. We use the word - applicable - to indicate the following proposition. The designations an interviewee mentally demarcates will inevitably be limited to the subject of a prospective or ongoing interview, as opposed to the interviewee's general knowledge. Later, we explain in detail how interviewees mentally determine applicable information items.

An illustration may assist in understanding the postulations just described. Consider the following fictitious scenario. An informant has discovered that a criminal network smuggles oxycodone into a prison using ambulances. The informant will be questioned in a routine

\footnotetext{
${ }^{1}$ Housekeeping notes: the phrases items of information and units of information are used synonymously throughout this article. We deviate from the standard presentation style of empirical articles. The present article is a hybrid that first presents a nascent theory and then proposes initial studies to examine the theory's core premise. We advise the reader to expect a more in-depth introduction than usual before the empirical aspect of the article.

${ }^{2}$ As in everyday communication, clarity can be subjective. What an interviewee considers clear and logical may not be perceived by another as such.
} 
MENTALLY DESIGNATING INFORMATION ITEMS IN INTERVIEWS

meeting. Let us assume that the criminal network is the usual reason for the meetings. Thus, the interviewer knows about the network but not the informant's recent discovery. We contend that the informant's discovery and, by extension, many subjects of interest could be mentally represented as a single information unit or multiple items-with varying degrees of completeness. Here, completeness refers to the full extent of an interviewee's knowledge on the subject. In the current example, the informant could mentally represent the discovery in any or all of the following formats. She could truly think that the interviewer might want to know any of the following things about the criminal network.

(z1) They smuggle oxycodone into a prison using ambulances.

(z2) They smuggle oxycodone into a prison.

(z3) They smuggle oxycodone.

(z4) They smuggle oxycodone using ambulances.

We use the preceding sentences to communicate the possible mental representations, in the present illustration, to the reader. In an actual instance, information units need not be or be thought of as complete sentences. We are positing that such representations-whatever their format or completeness - will embody what an interviewee envisions they could disclose. The word could is not trivial here. The interviewee may disclose or withhold the mentally identified information depending on further strategic considerations. Mentally designating information items is a crucial precursor to disclosure considerations and decisions, but this aspect of interviewees' cognitive process remains unexamined. This article aims to fill the flagged research gap.

In the current intelligence interviewing research paradigm, researchers motivate participants to disclose and withhold information (e.g., Dawson, 2015; Neequaye, 2018; Oleszkiewicz, 2016). Participants who assume the role of mock interviewees receive a twofold instruction. They are instructed not to share too little information because assisting the 
MENTALLY DESIGNATING INFORMATION ITEMS IN INTERVIEWS

interviewer may be beneficial. They are also told not to share too much information to avoid risks of disclosure. These instructions allow research studies to mimic the typical dilemma interviewees face in real intelligence interviews (see, e.g., Soufan, 2011). A recent review indicates that when responding to direct questions, the mock interviewee role leads participants to disclose some but not all the information they possess (Luke, 2021). We can infer from this finding that interviewees implement a honing process, at least intuitively, to determine the relevant information at their disposal. And they disclose their preferred items out of the lot. Put differently, interviewees mentally designate applicable information items and subsequently choose what to disclose.

The processes by which interviewees mentally itemize what they could disclose remains unknown. As a reminder, here, we do not mean the information an interviewee is necessarily willing to disclose or chooses to disclose. We mean: how an interviewee determines the germane information at their disposal. Subsequently, depending on the circumstances at hand, an interviewee may fully disclose or withhold the information altogether. One could also reveal partial bits of the information. Or the interviewee could replace the information with a false one and deceive the interviewer. Most research studies focus on these behaviors that interviewees enact after mentally designating applicable information items. No research endeavor has specified the mechanisms by which interviewees mentally designate applicable information units in the first place. To our knowledge, there is currently no concerted effort in that regard.

Understanding how interviewees mentally establish information item designations will expand current insights about the underpinnings of disclosure in intelligence interviews. Such knowledge allows the possibility to scrutinize another layer of influence: how interviewing approaches also impact the mental designation of information items to affect disclosure. This insight will add to existing research examining the processes that affect 
MENTALLY DESIGNATING INFORMATION ITEMS IN INTERVIEWS

disclosure. In short, this article explores how interviewees mentally determine the germane information at their disposal, which they may or may not disclose.

\section{Deciphering Information objectives and the Consequences Thereof}

Empirical and anecdotal evidence indicates that unyielding interviewees in investigative interviews employ various countermeasures to avoid cooperating with interviewers (Alison et al., 2014; Granhag et al., 2015; Soufan, 2011). For example, sometimes, such interviewees claim forgetfulness. We can thus assume that generally, unyielding interviewees intuitively estimate the subject of interest or an interviewer's information objectives to evade fully cooperating with such interests. By extension, it also stands to reason that cooperative interviewees similarly determine what the subject of interest is before cooperating by providing the commensurate useful information. We contend that, in intelligence interviews, like typical investigative interviews, interviewees try to understand what subjects are of interest to a prospective or current interviewer. From such understandings, interviewees mentally hone in on what information out of their general knowledge may be applicable.

\section{Relevance Theory as an Account of Construing Information Objectives}

Theorists in pragmatics have established that individuals decode communicators' utterances to understand the possible messages being relayed (Grice, 1989; McHoul, 1987). Consequently, a sender's utterances, whether verbal or non-verbal, serve as inputs guiding the receiver's comprehension. According to relevance theory, such inputs gain significance or relevance based on a receiver's evaluation that the input will contribute a worthwhile difference to their comprehension of the world (Sperber \& Wilson, 1995). These useful comprehensions include: reinforcing, modifying, or discarding prior understandings. More relevant inputs in that regard are likelier to be processed to a deeper degree - they will exert higher levels of influence on a receiver's comprehension (Wilson \& Sperber, 2002). 
MENTALLY DESIGNATING INFORMATION ITEMS IN INTERVIEWS

Importantly, individuals actively search for relevant inputs when deciphering a sender's meaning. Those inputs are typically interpreted within the context of the supposed interaction. The specific circumstances at hand contribute to the saliency of relevant inputs such that some inputs become more salient than others in different settings (Wilson \& Sperber, 2002).

The following illustration demonstrates the tenets of relevance theory just described. Imagine that you intend to watch a television show, Fun Psyc, scheduled to air at 19.00 on Channel UMO. At 18.55, you turn on your TV and see that the station is reporting an event they claim is breaking news: a highway motor accident. The input you have just received may lead you to conclude that Fun Psyc could be delayed. You may have to wait just a little longer, five minutes. Now consider the addition of further contextual information. For instance, the knowledge that Channel UMO's coverage of highway motor accidents usually lasts approximately one hour. Such contextual information may lead the input to gain relevance yielding further implications. For example, that Fun Psyc could be postponed, and you are better off making other plans.

We can think of an interviewer's solicitation attempts as information-seeking utterances or inputs. These inputs gain relevance based on an interviewee's estimation that an utterance contributes a worthwhile difference to understanding what the interviewer wants to $\mathrm{know}^{3}$. Consequently, more relevant inputs exert greater influence on an interviewee's comprehension of the objectives at hand. Interviewees then mentally organize the information they possess into item designations they believe correspond to the objective of those relevant information-seeking utterances. Recall the earlier example involving informing on a criminal network: mentally, the informant can subjectively determine the following. Out of the

\footnotetext{
${ }^{3}$ We use the terms information-seeking utterance and input in a broad sense. The terms include all the potential ways an interviewer may deliberately or inadvertently communicate their information objectives. Thus, solicitation attempts may include clear inquiries such as direct questions, vague solicitations such as a general request for an interview, or statements intended to influence the interviewee to make an inference about a supposed question.
} 
MENTALLY DESIGNATING INFORMATION ITEMS IN INTERVIEWS

information possessed, item z3 - they smuggle oxycodone-best matches the interviewer's perceived objective to discover the latest development in the network. The objective could be deciphered from the information-seeking utterance-have you discovered anything new about the network? Figure 1 illustrates the proposed sequence by which interviewees subjectively designate information units mentally.

\section{Figure 1}

The proposed sequence by which interviewees subjectively designate information items

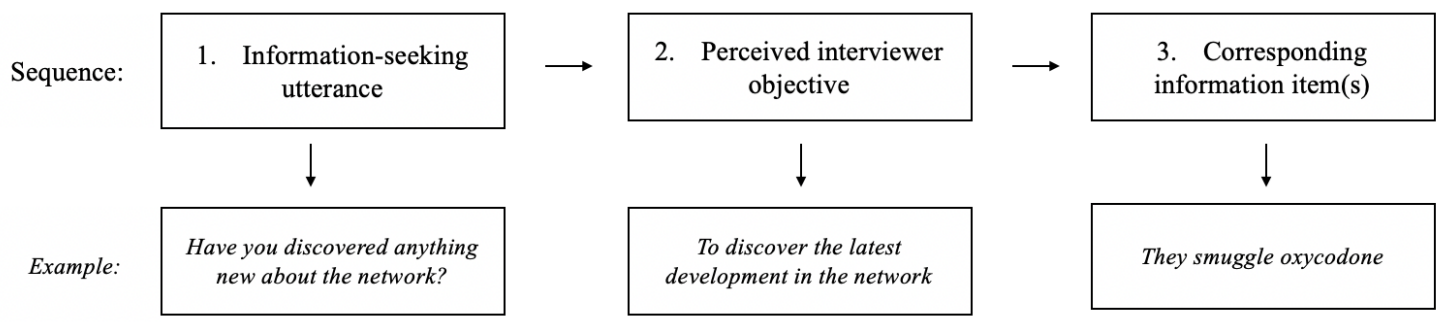

We contend that such systematization is a necessary first step, which subsequently undergoes any further processing that influences disclosure of the information. An example of such further processing could be managing the expected outcomes of disclosure by strategically sharing items that are ostensibly beneficial or innocuous to one's self-interests (Neequaye et al., 2018). Similar to navigating many social interactions, understanding information objectives, and mentally demarcating corresponding information units may recur throughout an interview. The interviewee stops mentally organizing when they exhaust the interviewer's information-seeking utterances with possible matching information items. The process restarts when the interviewee perceives novel utterances that require matching with commensurate information units. Such novel utterances include an interviewee's reinterpretation of existing utterances. 
MENTALLY DESIGNATING INFORMATION ITEMS IN INTERVIEWS

The reader should note that our use of the verb to organize, in the preceding paragraph, is to emphasize the following stipulation. Interviewees implicitly or explicitly try to make sense of the information they possess in relation to whatever they perceive an interviewer's information objectives to be. That is, the applicability of the information in the interview. An implicit attempt at determining such applicability could be forming a hypothesis about the interviewer's objectives. Then the interviewee might privately itemize the information that may fulfill those objectives. An explicit attempt could be asking the interviewer what their goals are. After such an implicit or explicit determination of what the interviewee considers the germane information, the interviewee may perform any of the following behaviors. They may disclose the appropriate information, provide incomplete information, decline to assist, or lie. We are not suggesting that the interviewee's information is necessarily unorganized in the sense that it is meaningless prior to an interview. Indeed, such information can be substantive to an interviewee in contexts apart from an interview-for example, when discussing the subject with a friend.

\section{Ensuing Predictions}

Exponents of relevance theory have noted that a receiver's deciphering of a sender's meaning is predominantly a non-demonstrative process (Wilson \& Sperber, 2002). Nevertheless, the natural results of such a process may provide opportunities to examine the mechanism's verisimilitude. Thus, the current research needs to offer testable predictions about the following question. Can the mental designation of information items be differentially manipulated by varying the characteristics of information-seeking utterances? Next, we explore such possibilities.

\section{The Clarity and Worthwhileness of Information-seeking Utterances}

It is well established that humans have limited cognitive resources. Hence, we are inclined to manage those resources most efficiently when making sense of stimuli in our 
MENTALLY DESIGNATING INFORMATION ITEMS IN INTERVIEWS

environment (Grier et al., 2003; Kahneman, 1973). In this view, relevance theory posits that we actively seek out clear and worthwhile inputs that we estimate will contribute to understanding a sender's message. Conversely, receivers usually avoid discerning senders' meanings using messages that they perceive as convoluted and provide little insight about what a sender means (Sperber \& Wilson, 1995). As such, utterances that one perceives to be clearer and more worthwhile are likelier to determine the meaning a receiver derives from the messages a sender transmits (see, e.g., Girotto et al., 2001). Consider the following illustration modeled after one described by (Wilson \& Sperber, 2002, p. 252). Jemima wants to ask Maria if Maria is interested in having lunch together. Jemima could truthfully communicate the request in any of the following ways.

1. Do you want to have lunch with me?

2. If drinking too much water is fatal, I am asking if you want to have lunch with me.

Utterance-1 and -2 are similar in one important sense: they both contain Jemima's request. Nonetheless, utterance-1 is arguably more comprehensible; it provides immediate insight into Jemima's request, and Maria requires little effort to understand it. Utterance-2, on the other hand, is convoluted because it is phrased as a declarative statement instead of a question. Additionally, Maria needs to establish whether drinking too much water is fatal before working out whether Jemima wants to have lunch. This example is quite artificial. Typically, the messages a sender transmits about a subject or multiple topics are not simultaneous. However, a receiver may have to contend with more than one message to understand a sender's meaning on a particular subject. According to relevance theory, when a receiver has several inputs to appraise, the receiver performs an intuitive comparison of the utterances at hand (Wilson \& Sperber, 2002). The receiver then processes and derives the sender's meaning by drawing on the inputs whose characteristics mostly resemble utterance-1 : inputs that require the least effort by the receiver to decipher what the sender means. 
MENTALLY DESIGNATING INFORMATION ITEMS IN INTERVIEWS

Drawing on the principles just described, we theorize that interviewees are likelier to more deeply process information-seeking utterances that they perceive to embody the following characteristics. (a) Clarity: that is, inputs whose substantive meaning are discernible with minimal effort. (b) Worthwhileness: these are utterances an interviewee estimates to contribute an essential difference to deciphering an interviewer's information objectives beyond what the interviewee currently knows. All things being equal, one can reasonably evaluate the worthwhileness of a message if the utterance is sufficiently clear. This premise leads us to offer a proposition similar to Wilson and Sperber's (2002). When multiple information-seeking utterances on a topic are adequately clear, the interviewee will use the messages judged to be the most worthwhile to decipher the interviewer's information objectives. Consequently, interviewees will tend to mentally organize the information they possess into item designations corresponding to information-seeking utterances perceived to be more worthwhile.

\section{Situating the Present Theory}

Existing research works on memory reporting in investigative interviewing are related to the subject matter of the present theory. Others have also examined interviewers' selection of question-types when strategizing to elicit information (e.g., Griffiths et al., 2011). For brevity's sake, we will focus the discussion here on arguably the most influential one to date: the cognitive interview (CI). The CI is an interviewing approach designed to enhance the memory and recall of eyewitnesses (see Fisher \& Geiselman, 1992 for an in-depth discussion). CI research addresses disclosure and non-disclosure at the broad or macro level. That is, what makes an interviewee report accurate details on a topic, assuming the interviewee is willing to report on the topic. The effect of questioning strategies is also an aspect at the macro level.

The present theory tackles interviewees' cognition at the micro-level: what happens before, for example, mnemonic devices or strategic question-types might improve the accuracy 
MENTALLY DESIGNATING INFORMATION ITEMS IN INTERVIEWS

of disclosure. The present theory explains how interviewees hear from a question the thing that is being questioned (see, also, McHoul, 1987). Hearing what is being questioned from a question is a pragmatic matter; here, the interviewee's problem is not yet an issue of memory and recall. Our contention is that hearing what a question is questioning-pragmatic considerations - is the precursor which mentally enters information items into play ${ }^{4}$ in an interview. Then assuming an interviewee has decided to be fully cooperative and disclose everything in question, memory enhancers may help the interviewee elaborate. A resistant interviewee may refrain from disclosing the item(s) a question enters into play. And a semicooperative interviewee may or may not disclose those items depending on further strategic considerations. For instance, a semi-cooperative interviewee could think: the thing being questioned is too risky to disclose or OK to reveal. The current theory proposes that the cooperative, the semi-cooperative, and the resistant interviewee first contend the same problem: what is the thing being questioned? Pragmatic considerations then mentally enter information-item designations into play. Those mental designations may then be affected by mnemonic devices like the CI or strategic considerations to determine disclosure or nondisclosure.

An illustration to further clarify the distinctions just described. Recall again the earlier example about the informant who has just discovered a criminal gang under investigation smuggles oxycodone into a prison using ambulances. The interviewee could truly think any of the following item designations might be informative.

(z1) They smuggle oxycodone into a prison using ambulances.

(z2) They smuggle oxycodone into a prison.

(z3) They smuggle oxycodone.

\footnotetext{
${ }^{4}$ We use the phrase enter into play to mean the interviewee mentally flagging an item designation, which they may or may not disclose.
} 
MENTALLY DESIGNATING INFORMATION ITEMS IN INTERVIEWS

(z4) They smuggle oxycodone using ambulances.

The interviewer interested in knowing about where the gang currently peddles narcotics could truly ask question- $i$ or $-i i$.

i. Have you discovered anything about the gang's narcotics operations?

ii. Have you discovered anything about where the gang peddles narcotics?

Arguably, question- $i i$ versus $-i$ is a higher-worthwhileness question in terms of the interviewer's objective to know where the gang peddles narcotics. Question-ii better specifies the interviewer's objective. What the present theory proposes is that a question form like question- $i i$ will enter the most pragmatic mental designation into play; here, z2 - they smuggle oxycodone into a prison. Then assuming the informant wants to disclose what they have mentally flagged, a memory-enhancing device like the CI might help the informant elaborate.

Note that a question form like question- $i$ is not necessarily better or worse than question- $i i$, as a strategic or tactical interviewing matter. An interviewer might deliberately employ question- $i$ suppose the interviewer wanted to conceal the objective to specifically discover where the gang peddles narcotics. Nonetheless, posing a question form like question$i$, which hardly specifies a clear objective, will enter a varied selection of item designations into play between different interviewees. One interviewee could truly think the interviewer wants to know z1. Another interviewee could also truly think the interviewer wants to know z3, and so forth. Assume an interviewee mentally selects $\mathrm{z} 3$ as their preferred pragmatic designation. Then a memory-enhancing device like the CI might help the interviewee elaborate. The point of note is this: pragmatic considerations first occur at the micro-level. Memory and strategic considerations then exert their influence at the macro-level. Thus, an interviewer could ask question- $i$, a low-worthwhileness question form, or a high-worthwhileness question form, question-ii, when implementing an interviewing approach like the CI. 
MENTALLY DESIGNATING INFORMATION ITEMS IN INTERVIEWS

The postulations of the present theory are well established in pragmatics (Grice, 1989; McHoul, 1987; Sperber \& Wilson, 1987; Vandierendonck \& Van Damme, 1988). However, because we have adapted those general principles to the niched context of intelligence interviewing, it is useful to subject our proposal to an empirical test.

\section{Empirically Examining Information Item Designation: Proof of Concept}

We will conduct two identical experiments to examine our theory that question-type influences mental designations and disposition affects utterance choices. As such, Study 1 will focus on mental designations and Study 2 on utterance choices.

In both studies, participants will assume the role of an intelligence source with one of two dispositions, intending to be cooperative or resistant when engaging with their interviewer. The interviewer will ask ten questions on various topics: the interviewees' discoveries about a criminal gang under investigation. Half of the questions will specify an objective (highworthwhileness condition), the other half will not specify a clear objective (low-worthwhileness condition).

How participants engage with the interviewer's questions will depend on the respective studies. In Study 1, the designation experiment, participants will not provide direct answers to the questions; instead, they will be instructed to indicate what they think the interviewer wants to know-about their discovery. In doing so, participants will flag their preferred mental designation of an information item. Then participants will rate their confidence that their preferred item designation is indeed what the interviewer wants to know. Conversely, participants in Study 2, the utterance experiment, will provide direct answers to the interviewer's questions. Participants will indicate what they want to say in response to the interviewer's questions. 
MENTALLY DESIGNATING INFORMATION ITEMS IN INTERVIEWS

Suppose the theory under contention has verisimilitude: then the subsequent predictions should receive support, notably the core predictions. Study 1 will examine Core Hypotheses 1a, 1b, and the Auxiliary Hypothesis. Study 2 will examine Core Hypotheses 2a and $2 \mathrm{~b}$.

The core of the present theory is that question worthwhileness predicts mental information-item designation, regardless of disposition. Thus-in Study 1 - the highworthwhileness questions should elicit a greater preference for item designations that pragmatically correspond to the interviewer's specified objective (Core Hypothesis-1a). That difference in preference should manifest irrespective of whether the participant is a cooperative or resistant source (Core Hypothesis-1b).

One auxiliary hypothesis of the present theory is that high- versus low-worthwhileness questions allow interviewees to better identify the interviewer's objective. That assumption implies that high-worthwhileness questions will allow the interviewee to be more certain about what the interviewer wants to know. Hence, the high- versus low-worthwhileness condition should elicit greater confidence that the corresponding preferred item designations are indeed the things the interviewer wants to know. That difference in preference should manifest irrespective of whether the participant is a cooperative or resistant source (Auxiliary Hypothesis)

In Study 2, which examines utterance, the disposition variable should determine participants' choices. Cooperative versus resistant sources should offer answers that pragmatically correspond to the interviewer's specified objective (Core Hypothesis-2a). Cooperative sources should want to assist the interviewer by disclosing the information the interviewer wants to know. Cooperative sources should be susceptible to the influence of highversus low worthwhileness questions; but resistant sources should not be susceptible (Core Hypothesis-2b). Resistant sources should refrain from providing pragmatic disclosures regardless of whether the interviewer asks high- or low-worthwhileness questions. Such 
MENTALLY DESIGNATING INFORMATION ITEMS IN INTERVIEWS

sources should want to refrain from assisting the interviewer irrespective of the extent to which the interviewer's question specifies an objective. Terrorist suspects who decline to cooperate abstain from disclosure using behaviors like refusing to comment or claiming to have a lack of memory (Alison et al., 2014).

\section{Method}

\section{Participants and Design}

Resource constraints determined our sample size choice; the analysis plan elaborates on our choice in relation to hypotheses testing. We will recruit English speaking participants via the Prolific Academic participant pool (estimated compensation $=£ 8 / \mathrm{hr}$.) We aim to remain with a maximum of $N=400$ participants (Age $\geq 18$ years, balanced across sex) after any data exclusions.

Our studies will examine how question-type and disposition differentially influence designation and utterance choices. For that purpose, we will conduct two experiments: Study $1(N=200)$ will focus on designation choices, and Study $2(N=200)$ on utterance choices. The experiments will employ the same design: 2 (Question-type: low- vs. high-worthwhileness; within-subjects) $\times 2$ (Disposition: cooperative vs. resistant; between-subjects).

Resource constraints determined our maximum sample size of 200 participants per experiment. But we conducted simulations to examine the level of precision the chosen sample size can provide, given our planned hypotheses tests. The simulations indicated that 200 participants per experiment sufficed to reach the precision we desire under two conditions: (1) a scenario including a wide range of estimated effect sizes using a random draw of response probabilities; and (2) a scenario where there is no effect. The Analysis Plan provides a detailed description.

\section{Procedure}


MENTALLY DESIGNATING INFORMATION ITEMS IN INTERVIEWS

Both experiments will employ a similar procedure and materials. Hence, it is necessary to preempt potential treatment diffusion regarding designation and utterance choices. We will conduct Studies 1 and 2 simultaneously, using the same web link to randomly assign prospective participants to the respective studies. This approach will ensure that prospective participants partake in either Study 1 or 2, not both. The experiments will be entirely online, and we will introduce them as studies about communication within a law enforcement context. A Regional Ethics Review Board in Sweden has indicated that this research design does not require a full ethics review (DNR: 812-12). The research adheres to the guidelines of the Swedish Research Council. Before commencing the research, participants will provide informed consent to the procedure and will receive a full debriefing upon completion. The appendix contains the full details of all the materials described in the procedure.

For the sake of conciseness, what follows describes the procedure for Studies 1 and 2. When necessary, we will highlight the differences in protocol between the studies

Phase 1: Intelligence-Source Role. By reading a short background story, participants will assume the role of a source who can gather information about a criminal gang. The story mimics the typical intelligence scenario whereby sources contend the possibility of disclosing information to an interviewer. Research studies usually employ background stories to create such source roles (e.g., Granhag et al., 2015). The story manipulates sources' dispositions in two ways. Half of the participants will assume a mindset to be a cooperative source when engaging with their interviewer, cooperative condition. The other half will assume the mindset of a resistant source when engaging with their interviewer. We will include a manipulation check to assess the efficacy of the disposition manipulation (see Appendix A).

We will not include a semi-cooperative condition for analytic reasons. Three disposition conditions will bring overly complex models to our hypothesis tests and obscure interpretation. Using the cooperative and resistant conditions is prudent because a semi- 
MENTALLY DESIGNATING INFORMATION ITEMS IN INTERVIEWS

cooperative disposition includes both cooperative and resistant mindsets. Thus, the present study will still contribute to understanding how semi-cooperative sources might behave when acting on either a cooperative or resistant disposition. Additionally, the present studies are initial ones in this line of research. For that reason, we believe it is prudent to employ a parsimonious design at this time; this issue is revisited in the analysis plan.

Phase 2: Decision-making Instructions. Next, participants will receive instructions on how to engage with the interviewer's questions. The instructions will depend on the study participants are randomly assigned to undergo. We have included an instructional manipulation check (IMC) to identify and exclude inattentive participants who fail the check (see Appendix B).

Study 1: Mental Designation. In this experiment, participants will receive instructions about indicating their preferred mental designation of an information item. Due to the nature of the theoretical propositions under examination, it is necessary to ensure that participants indicate their preferred pragmatic designation — and not the information they necessarily intend to disclose. Thus, the instructions will tell participants that upon receiving a question from their interviewer, they (i.e., participants) are to indicate what they think the interviewer wants to know-not necessarily what they intend to disclose.

Study 2: Utterance. In this experiment, the instructions will tell participants to provide direct answers to the interviewer's questions. Thus, participants will indicate what they want to say in response to each question.

Phase 3: Discoveries, Questions, and Decisions. Here, participants will undergo ten scenarios presented in random order. Appendix $\mathrm{C}$ contains all the material described here. In each scenario, participants will first make a discovery about the criminal gang under investigation. Each discovery will contain three parts that describe the details therein in ascending order: (a) bare minimum details; (b) medium details, comprising a new detail plus 
MENTALLY DESIGNATING INFORMATION ITEMS IN INTERVIEWS

the bare minimum; and (c) complete details, consisting of a new detail including the bare minimum and medium details. For example, you have noticed that the drug deals happen in the evening (bare minimum) when the workday ends (medium) at 18.00. (complete). Thus, each discovery will produce three legitimate information items, also describing the discovery's details in ascending order. For example,

the drug deals happen in the evening (bare minimum);

the drug deals happen in the evening when the workday ends (medium);

the drug deals happen in the evening when the workday ends at 18.00. (complete).

After each discovery, the interviewer will pose either a high- or low-worthwhileness question, five questions per condition. In the high-worthwhileness condition, the questions will specify an objective. Those questions will ask for a specific thing - the complete details about the discovery. Each question in the low-worthwhileness condition will be ambiguous: such questions will ask for anything about the discovery under investigation. Thus, the lowworthwhileness question could reasonably elicit the bare minimum, medium, or complete details, respectively.

How we invite participants to engage with each question will depend on the study to which participants are assigned.

Study 1: Mental Designation. In this experiment, participants will flag their preferred mental designation of an information item-what they think the interviewer wants to know. They will indicate that preference by choosing any of the three options describing their discovery's bare minimum, medium, or complete details. Participants will also be provided the option to indicate that they cannot determine what the interviewer wants to know.

Participants who select an information item will go on to provide two ratings, in random order, examining their confidence in their choice. A mandatory rating will directly ask participants how confident they are that their preference is indeed what the interviewer wants 
MENTALLY DESIGNATING INFORMATION ITEMS IN INTERVIEWS

to know ( $1=$ not confident at all, 5 = completely confident $)$. An optional rating will ask participants to stake a hypothetical bet that their preference is what the interviewer wants to know; the wager will be a percentage of their compensation $(0 \%=$ none of my compensation, $100 \%=$ all of my compensation).

Study 2: Utterance. In this experiment, participants will choose what they want to say in response to the interviewer's question. They will decide by choosing any of the three options describing their discovery's bare minimum, medium, or complete details. We will include the option to respond by choosing "no comment": suppose a participant wishes to remain silent regarding the interviewer's question. Here, participants who select an information item will not provide confidence ratings since confidence in utterance is not germane to our theory. Moreover, implementing the confidence assessment in Study 2 is likely to confuse participants about their main task.

Notes on Phase 3. A critic may argue that using multiple-choice options will limit participants' cognitive maps, restricting their potential mental designations and utterances. We disagree. The topics under each discovery are well-defined, making it highly unlikely that participants will generate mental designations outside each discovery's scope. Interlocutors typically restrict their discourse moves to the topic of discussion unless they wish to ask further questions or change topics (Roberts, 2012). The present study examines the mental designations and utterances elicited by questions under discussion, not how people ask further questions or change topics. Moreover, using multiple-choice options eliminates the possibility of coding errors that can arise from wrangling free-text data.

In both experiments, we will include four control questions to flag the data of inattentive participants (see Appendix D).

\section{Exclusion criteria}


MENTALLY DESIGNATING INFORMATION ITEMS IN INTERVIEWS

We will exclude the data of participants who fail the decision-making IMC and those who fail one control question, respectively.

\section{Analysis Plan}

We will examine the core hypotheses using Bayesian categorical regression models. The analysis will produce posterior distributions over parameters quantifying the probability of each possible parameter value given the data. We will report the posterior mean with the corresponding 95\% credible interval (95\%-CrI) and the 95\% Highest Density Interval (HDI). The $95 \%-\mathrm{CrI}$ is the range around the posterior mean within which the true value of the parameter lies with a probability of 0.95 . The HDI is identical to the $\mathrm{CrI}$ if the posterior is symmetric; if the posterior is asymmetric, the endpoints of both intervals may differ.

Following (Kruschke, 2014; Vasishth et al., 2018), we will define a region of practical equivalence (ROPE) to test whether we found evidence consistent with our predictions. The ROPE can be understood as a null region. We will reject the null hypothesis if the parameter's HDI falls outside of the null region. If the parameter's HDI overlaps with the null region and the sign is positive, we can reject a theory postulating a negative effect. If the sign is negative, we can reject a theory postulating a positive effect. If the parameter's HDI falls within the null region, we conclude that the data are consistent with "no effect" (not to say that we have proved that the null hypothesis is true). We will not conclude anything from our data when the ROPE lies entirely within the parameter's HDI. Furthermore, the ROPE will serve as a stopping rule for testing: Once decided on the null region, we aim to collect data until

a) the $95 \%$ HDIs of the parameters of interest are, at most, as wide as the null region, or

b) we reach our maximum participant sample size of 400 (200 per experiment) due to resource constraints.

Given our planned hypotheses tests, we conducted simulations to examine the level of precision the chosen sample size can provide. Our desired level of precision was that the width 
MENTALLY DESIGNATING INFORMATION ITEMS IN INTERVIEWS

of the coefficients' $95 \%$ HDIs should be equal to or smaller than 0.5 . The utterance experiment (Study 2) served as the benchmark because that study includes four relevant outcome levels: no comment, bare details, medium details, and complete details. The designation experiment (Study 1), on the other hand, involves three relevant outcome levels: bare details, medium details, and complete details. Hence, Study 2 versus Study 1 might require a larger sample size to achieve acceptable precision even though both studies employ a similar design.

We ran four models with simulated data (see supplemental materials: Rmarkdown documents DataSimulation 1 and DataSimulation 2). The simulations indicated that our hypotheses tests require a sample size greater than 150 participants per experiment. A model with 200 participants reached our desired precision for two different scenarios. DataSimulation 1: a wide range of estimated effect sizes using a random draw of response probabilities; DataSimulation 2: a scenario where there is no effect. One can access the simulations in exhaustive detail here: https://osf.io/7tgwe/.

\section{Model Specification}

\section{Fixed and Random Effects}

To predict designation (Model 1) and utterance (Model 2) preferences, we will fit two Bayesian categorical regression models using the R (R Core Team, 2020) package brms (Bürkner, 2017). The package provides an interface to fit Bayesian mixed models using Stan (Stan Development Team, 2017).

For Model 1, the variables disposition (cooperative vs. resistant) and question-type (high- vs. low-worthwhileness) will be included as predictors. We will run a model including an interaction of both predictors for exploratory purposes. The dependent variable will be the probability of choosing designations with medium information, complete information, or the option not to specify a designation over designations with bare information. Model 2 will include disposition as a main effect. And we will add question-type as a nested effect to 
MENTALLY DESIGNATING INFORMATION ITEMS IN INTERVIEWS

examine the effect of question type at the two levels of disposition (cooperative and resistant sources). The dependent variable will be the probability of choosing utterances with medium information, complete information, or no comment over utterances with bare information. For both Models 1 and 2, designations and utterances with bare information will be the reference category. Both models will include varying intercepts and slopes for participants and scenario items. We implement that inclusion assuming (1) the effect of question-type on designation and utterance choices varies between participant and scenario item, and (2) the effect of disposition on designation and utterance choices varies between scenario item.

\section{Priors}

For both models, we will use the same weakly regularizing priors, which allow a reasonably wide range of parameter values and penalize very extreme values. The priors for the by-designation and -utterance intercepts will be normal distributions with mean 0 and standard deviation 3. For fixed effects, normal priors with a mean of 0 and a standard deviation of 1 will be used. Random effects will be modeled as a correlation matrix and a vector of standard deviations. The standard deviations will be assigned half-normal priors with a mean of 0 and a standard deviation of 1 . For the correlation matrix, an $\operatorname{LKJ}(2)$ prior will be used such that smaller correlations are favored over extreme values such as +/- 1 (Sorensen et al., 2016, Stan Development Team, 2017). A prior-sensitivity analysis will be carried out to assess whether priors are dominating the posterior distribution. We will specifically contrast the aforementioned models with models having the following less conservative prior specifications.

Intercept $\mathrm{N}(0,10) /$ student_t $(3,0,2.5)$

Fixed effects $\mathrm{N}(0,1)$ / flat prior

Random effects sd Half Normal $(0,1) /$ student_t $(3,0,2.5)$

Correlation $\operatorname{LKJ}(2) / \operatorname{LKJ}(1)$

\section{Predictions}


MENTALLY DESIGNATING INFORMATION ITEMS IN INTERVIEWS

\section{Model 1: Designation}

We expect Core Hypothesis-1a to produce the following result. High-worthwhileness questions should elicit a preference for participants to choose designations with complete and medium information over designations with bare information, and designations with bare information over the option to not choose any designation. There should be no effect of disposition on the participants' item designation preferences (Core Hypothesis-1b).

\section{Model 2: Utterance}

We expect Core Hypothesis-2a to produce the following result. Cooperative versus resistant sources should more frequently choose utterances with complete and medium information as opposed to utterances with bare information or the no comment option. According to Core Hypothesis-2b, cooperative sources should be prone to the influence of high-versus low worthwhileness questions; but resistant sources should be uninfluenced by question-type (Core Hypothesis-2b).

\section{Summary of Predictions}

In all, the question-type versus disposition manipulation should influence participants' choices to a greater extent in Study 1 (the designation experiment). Conversely, the disposition versus question-type manipulation should influence participants' choices to a greater extent in Study 2 (the utterance experiment). The present theory will be supported if the data aligns with the pattern of results specified here.

\section{Region of Practical Equivalence (ROPE) and Model Comparison}

Due to the novelty of our study design, particularly the intelligence interviewing aspect, we were unable to find directly related studies. To our knowledge, no research has examined how question-worthwhileness and disposition differentially influence mental designation and utterance, much less with categorical regression. Some relevant studies include Lorson, Cummins, et al. (2021) and Lorson, Rohde, et al. (2021) that examined the effect of disposition 
MENTALLY DESIGNATING INFORMATION ITEMS IN INTERVIEWS

(i.e., cooperation) on utterance choices. That research found cooperation effects ranging from 0.54 to 1.68 in log-odds. Hence, for the predictor disposition, we will assume the following range of log-odds as ROPE: -0.25 to 0.25 (width of 0.5 ). We will specify the same ROPE [0.25 to 0.25$]$ for the predictor question-type and the interaction terms.

Note that our specified ROPE range is more conservative than the default ROPE range of $[-0.18,0.18]$ based on (Kruschke, 2014) and negligible effect sizes for behavioral sciences according to Cohen (1988). We hope that the present study will assist future studies in specifying effect sizes and ROPE specifications. Suppose we were to follow the recommended heuristic in (Dienes, 2021). In that case, we would have chosen the lower limit of the $95 \%$ CI of effects found in similar studies, for example, Lorson, et al. (2021). Then a negligible effect would be anything less extreme than 0.1 . However, given our resource constraints, we will not be able to reach a precision with HDIs equal to or smaller than 0.2 in range.

Suppose we decided to use the bottom limit of the $95 \%$ CI for the combined disposition effect from the studies mentioned earlier instead of examining the smallest possible effect (i.e., 0.54). In that case, based on a combined $95 \%$ CI of [0.24, 0.83$]$, we would yield a very similar ROPE as the one specified above $([-0.24,0.24]$ instead of $[-0.25,0.25])$. That observation leads us to believe our specified ROPE $[-0.25,0.25]$ is prudent at this time. That notwithstanding, we acknowledge that we run the risk of missing plausible effects that are smaller than the limits of our ROPE.

\section{Sampling Process}

Samples will be drawn from the posterior distributions of the model parameters using the NUTS sampler (Hoffman \& Gelman, 2014). We will run four sampling chains, each collecting 4,000 iterations, whereby the first 1,000 iterations will be disregarded as part of the warm-up phase leading to 12,000 iterations available for analysis. This sampling process will be the same for all models. 
MENTALLY DESIGNATING INFORMATION ITEMS IN INTERVIEWS

\section{Secondary Analysis: Auxiliary Hypothesis}

The auxiliary hypothesis will also be examined within the Bayesian framework. We will report the posterior mean and the $95 \%$ credible interval $(95 \%-\mathrm{CrI})$ and the probability that a given coefficient is greater than zero given the data and model. According to the auxiliary hypothesis, the high- versus low-worthwhileness condition should elicit greater confidence that corresponding preferred item designations are indeed the things the interviewer wants to know. Importantly, that difference in preference should manifest irrespective of disposition. Next follows the model specifications.

\section{Fixed and Random Effects}

We will examine participants' confidence ratings by fitting a mixed-effects Bayesian ordinal (cumulative) regression model (Model 3). Question-type and disposition will be included to predict confidence. The model will include varying intercepts and slopes for participants and scenario items, assuming that the effect of question-type on confidence ratings varies between participants and scenarios.

\section{Priors}

Again, we will use weakly regularizing priors, which will allow a reasonably wide range of parameter values and at the same time penalized very extreme values. The priors for the intercept will be normal distributions with mean 0 and standard deviation 3 . For both fixed effects, we will use normal priors with a mean of 0 and a standard deviation of 1 . Random effects will be modelled as a correlation matrix and a vector of standard deviations. The standard deviations will be assigned half-normal priors with a mean of 0 , and a standard deviation of 1 . For the correlation matrix, a $\operatorname{LKJ}(2)$ prior will be used such that smaller correlations are favoured over extreme values such as $+/-1$. A prior-sensitivity analysis will be carried out to see whether priors are dominating the posterior distribution. We will specifically 
MENTALLY DESIGNATING INFORMATION ITEMS IN INTERVIEWS

contrast the aforementioned model with a model having the following less conservative prior specifications:

Intercept student_t $(3,1.6,2.5)$

Fixed effects flat prior

Random effects sd student_t $(3,0,2.5)$

Correlation $\operatorname{LKJ}(1)$

\section{Second Confidence Measure: Willingness to Bet}

We included a second confidence measure to examine the auxiliary hypothesis: the willingness of participants to place a bet that their preference is what the interviewer wants to know. Similar to the confidence ratings, high- versus low-worthwhileness questions are predicted to increase the probability of betting, independent of disposition. We will examine the prediction using a mixed-effects Bayesian logistic regression model (Model 4).

Fixed and Random Effects. Question-type and disposition will be included to predict the probability of betting. The model will include varying intercepts and slopes for participants and scenarios items, assuming that the effect of question type on the participants' confidence varies between participant and scenario items.

Priors. Again, we will use weakly regularizing priors, which allows a reasonably wide range of parameter values and penalizes very extreme values. The prior for the intercept is a normal distribution with mean 0 and standard deviation 3. For fixed effects, normal priors with a mean of 0 and a standard deviation of 1 will be used. Random effects will be modelled as a correlation matrix and a vector of standard deviations. The standard deviations will be assigned half-normal priors with a mean of 0 , and a standard deviation of 1 . For the correlation matrix, a LKJ(2) prior will be used such that smaller correlations are favoured over extreme values such as +/- 1. A prior-sensitivity analysis will be carried out to see whether priors are dominating the posterior distribution. We will specifically contrast the aforementioned model with a model having the following less conservative prior specifications: 
MENTALLY DESIGNATING INFORMATION ITEMS IN INTERVIEWS

Intercept $\mathrm{N}(0,10) /$ student_t $\mathrm{t}(3,0,2.5)$

Fixed effects $\mathrm{N}(0,1)$ / flat prior

Random effects sd Half Normal $(0,1) /$ student_t $(3,0,2.5)$

Correlation LKJ(2)/ LKJ(1) 


\section{MENTALLY DESIGNATING INFORMATION ITEMS IN INTERVIEWS}

\section{Study Design Template}

Notes:

1. We will code the outcome measure (information items) as follows (with 'bare' as reference level); the coding will be the same for all the relevant analyses:

$\begin{array}{llll} & \text { complete } & \text { medium } & \text { no comment } \\ \text { bare } & 0 & 0 & 0 \\ \text { complete } & 1 & 0 & 0 \\ \text { medium } & 0 & 1 & 0 \\ \text { no comment } & 0 & 0 & 1\end{array}$

1. Sampling plan and test sensitivity rationale: We aim to include a minimum of $N=400$ participants, $N=200$ per study. Resource constraints and the lack of previous research (to precisely estimate a Region of Practical Equivalence [ROPE]) determined our sample size choice. For Studies 1 and 2, it holds that we will not conclude anything from our data when the ROPE lies entirely within the parameter's HDI.

2. Theory that could be shown wrong by outcomes: This research is a first test of the theory outlined in the article. Thus, we cannot disprove the theory, at least not with certainty at this time. And we cannot rule out plausible effects that are smaller than the limits of our ROPE. Nonetheless, supporting or rejecting the respective core hypotheses will count for and against the theory's corresponding postulations. This approach will assist in potentially refining the theory and developing replication studies.

Table X1

Study 1 Designation Experiment

\begin{tabular}{|c|c|c|c|}
\hline Hypothesis & Model I & Analysis & Predictions \\
\hline $\begin{array}{l}\text { Core hypothesis 1a } \\
\text { High-worthwhileness questions } \\
\text { should elicit a greater preference for } \\
\text { item designations that pragmatically } \\
\text { correspond to the interviewer's } \\
\text { specified objective. }\end{array}$ & $\begin{array}{l}\text { brm }(\text { Response } \sim \text { Disposition + QuType }+ \\
\text { (QuType | SubjectID) + (Disposition + QuType } \\
\text { | Context) } \\
\text { Contrast coding for Model I: } \\
\text { Question Type: } \\
\quad \text { high-worthwhileness: } 1 \\
\quad \text { low-worthwhileness: }-1\end{array}$ & $\begin{array}{l}\text { To test this hypothesis, we will investigate } \\
\text { whether there is a main effect of Question } \\
\text { Type on the log-odds of choosing } \\
\text { complete/medium/no comment } \\
\text { designations over bare information } \\
\text { designations. }\end{array}$ & $\begin{array}{l}\mathrm{P}(\text { designation }=\text { complete/medium })> \\
\mathrm{P}(\text { designation }=\text { bare }) \\
\text { The Question Type parameters' HDI - for } \\
\text { both } \mathrm{P}(\text { complete }>\text { bare }) \text { and } \mathrm{P}(\text { medium }> \\
\text { bare }) \text { - should lie outside the ROPE and } \\
\text { have a positive sign for high } \\
\text { worthwhileness questions (which are } \\
\text { coded as } 1) .\end{array}$ \\
\hline
\end{tabular}




\begin{tabular}{|c|c|c|c|}
\hline & \multirow[t]{2}{*}{$\begin{array}{l}\text { Disposition: } \\
\qquad \begin{array}{l}\text { cooperative: } 1 \\
\text { resistant: }-1\end{array} \\
\text { The model for Study } 1 \text { will only include the } \\
\text { two predictors Question Type and Disposition } \\
\text { and no interaction term. A model including an } \\
\text { interaction term will be run for exploratory } \\
\text { purposes. }\end{array}$} & & $\begin{array}{l}\mathrm{P}(\text { designation }=\text { No comment })> \\
\mathrm{P}(\text { designation }=\text { bare }) \\
\text { The Question Type parameter's HDI } \\
\text { should lie outside the ROPE and should } \\
\text { have a negative sign for high } \\
\text { worthwhileness questions (which are } \\
\text { coded as } 1) .\end{array}$ \\
\hline $\begin{array}{l}\text { Core hypothesis } \mathbf{1 b} \\
\text { There should be no effect of } \\
\text { Disposition on the participants' item } \\
\text { designation preferences. }\end{array}$ & & $\begin{array}{l}\text { To test this hypothesis, we will investigate } \\
\text { whether there is a main effect of } \\
\text { Disposition on the log-odds of choosing } \\
\text { high/medium/no comment designations } \\
\text { over bare information designations. }\end{array}$ & $\begin{array}{l}\text { All the Disposition parameter's HDIs are } \\
\text { predicted to fall within the null region, } \\
\text { such that we conclude that the data are } \\
\text { consistent with "no effect" of Disposition } \\
\text { (not to say that we have proven that the } \\
\text { null hypothesis is true). }\end{array}$ \\
\hline
\end{tabular}

\section{Table X2}

Study 2 Utterance Experiment

\begin{tabular}{|c|c|c|c|}
\hline Hypothesis & Model II & Analysis & Predictions \\
\hline $\begin{array}{l}\text { Core hypothesis } 2 \text { a } \\
\text { Cooperative sources should more } \\
\text { frequently choose utterances with } \\
\text { complete/medium informativeness } \\
\text { as opposed to bare } \\
\text { informativeness or the no } \\
\text { comment option than resistant } \\
\text { sources. }\end{array}$ & $\begin{array}{l}\text { brm(Response } \sim \text { Disposition/QuType + (QuType | } \\
\text { SubjectID) + (Disposition/ QuType | Context) } \\
\text { Contrast coding for Model II: } \\
\text { Question Type: } \\
\quad \text { high-worthwhileness: } 0.5 \\
\quad \text { low-worthwhileness: }-0.5 \\
\text { Disposition: } \\
\quad \text { cooperative: } 0.5 \\
\quad \text { resistant: }-0.5 \\
\text { Parameter estimated: } \\
\text { complete_Intercept }\end{array}$ & $\begin{array}{l}\text { To test this hypothesis, we will } \\
\text { investigate whether there is a main } \\
\text { effect of Disposition on the log-odds } \\
\text { of choosing complete/medium/no } \\
\text { information utterances over bare } \\
\text { information utterances, holding the } \\
\text { predictor Question Type constant. }\end{array}$ & $\begin{array}{l}\mathrm{P}(\text { utterance }=\text { complete/medium })> \\
\mathrm{P}(\text { utterance }=\text { bare }) \\
\text { The Disposition parameter's HDI }- \text { for } \\
\text { both } \mathrm{P}(\text { complete }>\text { bare }) \text { and } \mathrm{P}(\text { medium }> \\
\text { bare }) \text { - should lie outside the ROPE and } \\
\text { should have a positive sign for } \\
\text { cooperative sources. } \\
\mathrm{P}(\text { utterance = no comment })>\mathrm{P}(\text { utterance } \\
=\text { bare }) \\
\text { The Disposition parameter's HDI should } \\
\text { lie outside the ROPE and should have a } \\
\text { negative sign for cooperative sources. }\end{array}$ \\
\hline
\end{tabular}




\begin{tabular}{|c|c|c|c|}
\hline & \multirow[b]{2}{*}{$\begin{array}{l}\text { complete_Disposition1 } \\
\text { complete_Dispositioncooperative:QuType1 } \\
\text { complete_Dispositionresistant:QuType1 } \\
\text { medium_Intercept } \\
\text { medium_Disposition1 } \\
\text { medium_Dispositioncooperative:QuType1 } \\
\text { medium_Dispositionresistant:QuType1 } \\
\text { nocomment_Intercept } \\
\text { nocomment_Disposition1 } \\
\text { nocomment_Dispositioncooperative:QuType1 } \\
\text { nocomment_Dispositionresistant:QuType1 } \\
\text { This model tests the main effect of Disposition } \\
\text { (Disposition1) averaged over the levels of } \\
\text { Question Type, and the effect of Question Type } \\
\text { within the group of cooperative sources } \\
\text { (Dispositioncooperative:QuType1) and resistant } \\
\text { sources (Dispositionresistant:QuType1). Thus, the } \\
\text { model tests whether the two levels of Question } \\
\text { Type differ for cooperative versus resistant } \\
\text { sources. }\end{array}$} & & \\
\hline $\begin{array}{l}\text { Core hypotheses } \mathbf{2 b} \\
\text { Only cooperative sources as } \\
\text { opposed to resistant sources } \\
\text { should be affected by the predictor } \\
\text { Question Type. } \\
\rightarrow \text { Cooperative sources should be } \\
\text { influence by the predictor } \\
\text { Question Type. } \\
\rightarrow \text { Resistant sources should not be } \\
\text { influenced by the predictor } \\
\text { Question Type. }\end{array}$ & & $\begin{array}{l}\text { To test hypothesis } 2 \mathrm{~b} \text { we will } \\
\text { investigate whether there is a simple } \\
\text { effect of Question Type on the log- } \\
\text { odds of choosing high/medium/no } \\
\text { information utterances over bare } \\
\text { information utterance for cooperative } \\
\text { versus resistant sources. }\end{array}$ & $\begin{array}{l}\text { Cooperative Group } \\
\text { (Dispositioncooperative:QuType): } \\
\text { P(utterance = complete/medium/no } \\
\text { comment) > P(utterance = bare) } \\
\text { The Question Type parameter's HDI } \\
\text { should lie outside the ROPE such that } \\
\text { there is a difference between seeing a } \\
\text { high- versus low-worthwhileness } \\
\text { question for cooperative sources. } \\
\text { Resistant Group } \\
\text { (Dispositionresistant:QuType1) } \\
\text { All the Question Type parameter's HDIs } \\
\text { are predicted to fall within the null } \\
\text { region, such that we conclude that the } \\
\text { data are consistent with "no effect" of } \\
\text { Question Type for resistant sources (not } \\
\text { to say that we have proven that the null } \\
\text { hypothesis is true). }\end{array}$ \\
\hline
\end{tabular}

\section{Auxiliary Hypothesis: Confidence in decisions (Study 1)}

\section{Table X3}

Confidence Ratings

Output (5-point Likert Scale): 'not confident at all', 'slightly confident', 'somewhat confident', 'fairly confident', 'completely confident' 


\begin{tabular}{|c|c|c|c|}
\hline Hypothesis & Model III (Ordinal cumulative modeL) & Analysis & Predictions \\
\hline $\begin{array}{l}\text { The high- versus low-worthwhileness } \\
\text { condition should elicit greater confidence, } \\
\text { but Disposition should not influence the } \\
\text { ratings. }\end{array}$ & $\begin{array}{l}\text { brm(Likert } \sim \text { QuType + Disposition + } \\
\text { (QuType | SubjectID) + (QuType + } \\
\text { Disposition | Context) } \\
\text { Contrast coding for Model I: } \\
\text { Question Type: } \\
\quad \text { high-worthwhileness: } 1 \\
\text { low-worthwhileness: }-1 \\
\text { Disposition: } \\
\text { cooperative: } 1 \\
\text { resistant: }-1\end{array}$ & $\begin{array}{l}\text { To test this hypothesis, we will investigate } \\
\text { whether there is a main effect of Question } \\
\text { Type on the confidence ratings. }\end{array}$ & $\begin{array}{l}\text { The Question Type parameter's HDI should } \\
\text { lie outside the ROPE and should have a } \\
\text { positive sign for high worthwhileness } \\
\text { questions (which are coded as 1). } \\
\text { The Disposition parameter's HDI is } \\
\text { predicted to fall within the null region, such } \\
\text { that we conclude that the data are consistent } \\
\text { with "no effect" of Disposition on the } \\
\text { confidence ratings (not to say that we have } \\
\text { proven that the null hypothesis is true). }\end{array}$ \\
\hline
\end{tabular}

\section{Table X4}

Willingness to bet

Output: willingness to bet, 'yes' (1), 'no' (0)

\begin{tabular}{|l|l|l|l|}
\hline \multicolumn{1}{|c|}{ Hypothesis } & Model IV (Ordinal Cumulative model) & \multicolumn{1}{|c|}{ Analysis } & \multicolumn{1}{|c|}{ Predictions } \\
\hline $\begin{array}{l}\text { High- versus low-worthwhileness questions } \\
\text { should increase the probability of betting, } \\
\text { independent of disposition. }\end{array}$ & $\begin{array}{l}\text { lie outside the ROPE and should have a } \\
\text { positive sign for high worthwhileness } \\
\text { questions (which are coded as 1). } \\
\mid \text { SubjectID) + (QuType + Disposition } \mid \\
\text { Context) }\end{array}$ & $\begin{array}{l}\text { To test this hypothesis, we will investigate } \\
\text { whether there is a main effect of Question } \\
\text { Type on the participants' willingness to bet. }\end{array}$ & $\begin{array}{l}\text { The Disposition parameter's HDI is } \\
\text { predicted to fall within the null region, such } \\
\text { that we conclude that the data are consistent } \\
\text { with "no effect" of Disposition on the } \\
\text { confidence ratings (not to say that we have } \\
\text { proven that the null hypothesis is true). }\end{array}$ \\
\hline
\end{tabular}


MENTALLY DESIGNATING INFORMATION ITEMS IN INTERVIEWS

\section{References}

Alison, L., Alison, E., Noone, G., Elntib, S., Waring, S., \& Christiansen, P. (2014). Whatever you say, say nothing: Individual differences in counter interrogation tactics amongst a field sample of right wing, AQ inspired and paramilitary terrorists. Personality and Individual Differences, 68, 170-175. https://doi.org/10.1016/j.paid.2014.04.031

Brandon, S. E. (2014). Towards a Science of Interrogation. Applied Cognitive Psychology, 28(6), 945-946. https://doi.org/10.1002/acp.3090

Bürkner, P.-C. (2017). Advanced Bayesian Multilevel Modeling with the R Package brms. The R Journal, 10(1), 395-411.

Cohen, J. (1988). Statistical Power Analysis for the Behavioral Sciences (2nd ed.). Routledge. https://doi.org/10.4324/9780203771587

Dawson, E. C. (2015). Improving Investigative Interviews: Facilitating Disclosure of Information through Implicit Means [Graduate Center, City University of New York]. https://academicworks.cuny.edu/gc_etds/900

Dienes, Z. (2021). Obtaining Evidence for No Effect. Collabra: Psychology, 7(1), 28202. https://doi.org/10.1525/collabra.28202

Fisher, R. P., \& Geiselman, R. P. (1992). Memory Enhancing Techniques for Investigative Interviewing. Springfield, Illinois: Charles C. Charles C. Thomas.

Girotto, V., Kemmelmeier, M., Sperber, D., \& van der Henst, J.-B. (2001). Inept reasoners or pragmatic virtuosos? Relevance and the deontic selection task. Cognition, 81(2), B69-B76. https://doi.org/10.1016/S0010-0277(01)00124-X

Granhag, P. A., Montecinos, S. C., \& Oleszkiewicz, S. (2015). Eliciting intelligence from sources: The first scientific test of the Scharff technique. Legal and Criminological Psychology, 20(1), 96-113. https://doi.org/10.1111/lcrp.12015

Grice, H. P. (1989). Studies in the Way of Words. Harvard University Press. 
MENTALLY DESIGNATING INFORMATION ITEMS IN INTERVIEWS

Grier, R. A., Warm, J. S., Dember, W. N., Matthews, G., Galinsky, T. L., Szalma, J. L., \& Parasuraman, R. (2003). The Vigilance Decrement Reflects Limitations in Effortful Attention, Not Mindlessness. Human Factors, 45(3), 349-359. https://doi.org/10.1518/hfes.45.3.349.27253

Griffiths, A., Milne, B., \& Cherryman, J. (2011). A Question of Control? The Formulation of Suspect and Witness Interview Question Strategies by Advanced Interviewers. International Journal of Police Science \& Management, 13(3), 255-267. https://doi.org/10.1350/ijps.2011.13.3.219

Hoffman, M. D., \& Gelman, A. (2014). The No-U-Turn Sampler: Adaptively Setting Path Lengths in Hamiltonian Monte Carlo. Journal of Machine Learning Research, 15, $1593-1623$.

Hope, L., Gabbert, F., \& Fisher, R. P. (2011). From laboratory to the street: Capturing witness memory using the Self-Administered Interview. Legal and Criminological Psychology, 16(2), 211-226. https://doi.org/10.1111/j.2044-8333.2011.02015.x

Kahneman, D. (1973). Attention and Effort. Englewood Cliffs.

Kruschke, J. (2014). Doing Bayesian Data Analysis: A Tutorial with R, JAGS, and Stan. Academic Press.

Lorson, A., Cummins, C., \& Rohde, H. (2021). Strategic Use of (Un)certainty Expressions. Frontiers in Communication, 6. https://www.frontiersin.org/article/10.3389/fcomm.2021.635156

Lorson, A., Rohde, H., \& Cummins, C. (2022). The strategic and epistemic properties of 'know’. PsyArXiv. https://doi.org/10.31234/osf.io/8zb37

Luke, T. J. (2021). A meta-analytic review of experimental tests of the interrogation technique of Hanns Joachim Scharff. Applied Cognitive Psychology, acp.3771. https://doi.org/10.1002/acp.3771 
MENTALLY DESIGNATING INFORMATION ITEMS IN INTERVIEWS

McHoul, A. W. (1987). Why there are no guarantees for interrogators. Journal of Pragmatics, 11(4), 455-471. https://doi.org/10.1016/0378-2166(87)90089-0

Neequaye, D. A. (2018). Eliciting information in intelligence interviews through priming: An examination of underlying mechanisms [Department of Psychology, University of Gothenburg]. https://gupea.ub.gu.se/handle/2077/57528

Neequaye, D. A., Luke, T. J., \& Kollback, K. (2018). Managing Disclosure Outcomes in Intelligence Interviews. PsyArXiv. https://doi.org/10.31234/osf.io/tfp2c

Oleszkiewicz, S. (2016). Eliciting human intelligence a conceptualization and empirical testing of the Scharff-technique [Department of Psychology, University of Gothenburg]. http://hdl.handle.net/2077/41567

R Core Team. (2020). R: A Language and Environment for Statistical Computing [Computer software manual]. Vienna, Austria. Retrieved from Mhttps://www.R-project.org/

Roberts, C. (2012). Information structure in discourse: Towards an integrated formal theory of pragmatics. Semantics and Pragmatics, 5. https://doi.org/10.3765/sp.5.6

Sorensen, T., Hohenstein, S., \& Vasishth, S. (2016). Bayesian linear mixed models using Stan: A tutorial for psychologists, linguists, and cognitive scientists. The Quantitative Methods for Psychology, 12(3), 175-200. https://doi.org/10.20982/tqmp.12.3.p175

Soufan, A. (2011). The Black Banners: The Inside Story of 9/11 and the War Against alQaeda. W. W. Norton \& Company.

Sperber, D., \& Wilson, D. (1987). Précis of Relevance: Communication and Cognition. Behavioral and Brain Sciences, 10(4), 697-710. https://doi.org/10.1017/S0140525X00055345

Sperber, D., \& Wilson, D. (1995). Relevance: Communication and cognition (2nd ed). Blackwell Publishers. 
MENTALLY DESIGNATING INFORMATION ITEMS IN INTERVIEWS

Stan Development Team. (2017). Stan modeling language users guide and reference manual, version 2.17.0 [Computer software manual]. Retrieved from Mhttp://mc-stan.org/

Vandierendonck, A., \& Van Damme, R. (1988). Schema anticipation in recall: Memory process or report strategy? Psychological Research, 50(2), 116-122. https://doi.org/10.1007/BF00309211

Vasishth, S., Mertzen, D., Jäger, L. A., \& Gelman, A. (2018). The statistical significance filter leads to overoptimistic expectations of replicability. Journal of Memory and Language, 103, 151-175. https://doi.org/10.1016/j.jml.2018.07.004

Vehtari, A., Gelman, A., \& Gabry, J. (2017). Practical Bayesian model evaluation using leave-one-out cross-validation and WAIC. Statistics and Computing, 27(5), 14131432. https://doi.org/10.1007/s11222-016-9696-4

Vehtari, A., Simpson, D., Gelman, A., Yao, Y., \& Gabry, J. (2015). Pareto Smoothed Importance Sampling. https://arxiv.org/abs/1507.02646v7

Wilson, D., \& Sperber, D. (2002). Relevance theory. In Handbook of Pragmatics. Blackwell. 


\section{$\underline{\text { Appendices }}$}

\section{Introduction}

This study is about communication within a law enforcement context. You will read some fictional scenarios assuming the role of the main character. Then you will answer some questions about each scenario. Most of this study involves reading. So, please read the scenarios and instructions carefully because understanding them is crucial. We have included questions to check if you read and answered questions with your full attention.

The entire study will take approximately XXX minutes to complete. You will receive a compensation of XXX for participating once the study is over.

\section{Appendix $A$}

\section{Disposition manipulations}

Imagine that you are one of the owners of a restaurant in town; you also work at this restaurant, which overlooks a big park. You and your colleagues have a good picture of what goes on in the park. It is well known among the restaurant staff that a narcotics-dealing gang called KET22 operates in the park. Recently, a police-contact approached you and your colleagues to provide information about the gang if you discovered anything. The police-contact mentioned that none of you are obliged to give any information. $<$ Dispositional variations begin here $>$

Cooperative: However, KET22 disrupts your business at the restaurant. So, it is in your best interest to assist the police in their investigations to eliminate the gang. Then your business can grow.

Resistant: The police-contact does not know this: but because you (personally) came into some financial troubles, you occasionally supply narcotics to customers at the restaurant on the gang's behalf. If the gang gets busted, you are very likely to get in trouble too. You only agreed to meet with the police-contact to avoid suspicion.

\section{Manipulation check (disposition)}

Now you know your character or the role you are to play in this study. Suppose you were to make discoveries that could get KET22 busted, and the police-contact asked you about those discoveries. How would you engage with the interviewer?

- I will lie to ensure that I hide what I know. (-1)

- I will keep silent and not respond to the question. (0)

- I will reveal some of my discoveries, not everything I know. (1)

- I will reveal what I know. (2)

\section{Appendix B}

\section{Introduction to scenarios}

In the next phase of the study, you will be placed in various scenarios where you will make various discoveries about KET22, the gang under investigation. After each discovery, you will receive a question from the police contact about the discovery. $<$ Variations between Studies 1 and 2 begin here $>$ 
Study 1 (Designation experiment):-

Your task in the upcoming phase is to indicate what you think the police-contact WANTS TO KNOW about your discovery based on the police-contact's question. The task is NOT about indicating what you will necessarily say in response to the question.

Your task is to indicate what you think the police-contact wants to know based on the police-contact's question!

We have included other questions to check if you read and answered the questions with your full attention.

\section{Study 2 (Utterance experiment):-}

Your task in the upcoming phase is to indicate WHAT YOU WANT TO SAY in response to the police-contact's question.

We have included other questions to check if you read and answered the questions with your full attention.

\section{Instructional manipulation check (Study 1 and 2)}

Passing or failing this IMC will depend on whether a participant undergoes Study 1 or 2.

What is TRUE about your main task in the upcoming phase?

- My task is to indicate what I think the police-contact wants to know based on the policecontact's question.

- My task is to indicate what I want to say in response to the police-contact's question.

Next follows the scenarios.

\section{Appendix $C$}

Participants will be randomly assigned to one of two lists to facilitate randomization. And the contents of the lists will be presented in random order in both the utterance and designation conditions.
List 1
List 2
Scenario 1a Scenario $1 \mathrm{~b}$
Scenario $2 b \quad$ Scenario $2 a$
Scenario 3a Scenario 3b
Scenario 4b Scenario 4a
Scenario 5a Scenario 5b
Scenario 6b Scenario 6a
Scenario 7a Scenario 7b
Scenario 8b Scenario 8a
Scenario 9a Scenario 9b
Scenario 10b Scenario 10a 
MENTALLY DESIGNATING INFORMATION ITEMS IN INTERVIEWS

Before presenting the information items in each scenario, the designation and utterance conditions will include the following prompts, respectively.

- Select what you think the interviewer wants to know. (Study 1, Designation Experiment)

- Select what you want to say. (Study 2, Utterance Experiment)

Apart from the information items, the designation and utterance experiments will include the following options, respectively

- I cannot determine what the interviewer wants to know (Study 1, Designation Experiment).

- No comment (Study 2, Utterance Experiment)

\section{Scenarios}

a. High worthwhileness questions marked with green highlight

b. Low worthwhileness questions marked with yellow highlight

1. One day after work, on your bus ride home, you recognized one of the KET22 members. You were sitting just behind him, and he was talking on the phone. He tried to be quiet, but you heard him say: "It is better to sell the off-brand green-star oxycodone."

a. Have you discovered the particular brand of narcotics KET22 sells?

b. Have you discovered anything about the gang's narcotics sales lately?

i. They sell oxycodone.

ii. They sell green-star oxycodone.

iii. They sell the off-brand green-star oxycodone.

2. Your curiosity has led you to pay more attention to KET22's drug dealings. You've discovered that the gangsters usually deal their drugs to customers in the evenings when the workday ends, around 18.00.

a. Do you have an idea of what specific time the gangsters deal drugs to customers?

b. Do you have any information about when the gang deals drugs?

i. The drug deals happen in the evening.

ii. The drug deals happen in the evening when the workday ends.

iii. The drug deals happen in the evening when the workday ends at 18.00.

3. You always come to work earlier than your colleagues because you supervise the cleaners. You've realized that the KET22 gangsters usually arrive shortly after you in a blue Nissan Qashqai. By paying more attention, you've memorized the license plate number: FBT038.

a. Do you know the full details about the vehicle the KET22 gangsters usually arrive in at the park?

b. Do you have any information about KET22's transportation in the park?

i. They usually arrive in a Nissan.

ii. They usually arrive in a Nissan Qashqai.

iii. They usually arrive in a Nissan Qashqai, license plate number FBT038.

4. During one of your short breaks at work, you decided to enjoy some sunshine. So, you went to the edge of the park where there are benches. As you approached, you saw a rowdy group at one of the benches, and you chose the bench furthest away from them. The group was talking about how to contact KET22 to buy narcotics. They said 
MENTALLY DESIGNATING INFORMATION ITEMS IN INTERVIEWS

customers could make contact by sending a text message containing a lion emoji to any KET22 phone number.

a. Have you made observations about exactly how customers contact KET22 to buy narcotics?

b. Have you made any observations about KET22's customers?

i. Customers make contact by phone.

ii. Customers make contact by sending a text message.

iii. Customers make contact by sending a text message containing a lion emoji.

5. Lately, you have noticed a particular spot at the park where the KET22 gangsters deal drugs. The spot is one of the park's exits, EXIT 7F. All the exits are located at different edges of the park, but 7F is rather discreet.

a. Have you spotted the exact location at the park where KET22 deals drugs?

b. Have you spotted anything about where KET22 deals drugs?

i. The gangsters deal at the edge of the park.

ii. The gangsters deal at an exit at the edge of the park.

iii. The gangsters deal at EXIT 7F, a discreet location at the edge of the park.

6. From your observations of the park, it seems that the gangsters take shifts selling their narcotics. Their rotations include five (5) gangsters, two (2) sell, and three (3) are lookouts.

a. Have you discovered the specific methods the gangsters use when selling narcotics?

b. Have you observed anything about the gang's drug sales?

i. They take shifts selling narcotics.

ii. They take shifts selling narcotics, five gangsters at a time.

iii. They take shifts selling narcotics, five (5) gangsters at a time; two (2) sell, and three (3) are lookouts.

7. On your way home after work, you saw that some KET22 gangsters were arguing. It was around 19.00 on Monday. From what you heard, the argument was about whether to sell a high dose of drugs to a customer.

a. Have you caught the contents of particular interactions between the gang members lately?

b. Have there been any developments with the gang members lately?

i. There was an argument between some gang members.

ii. On Monday at 19.00, there was an argument between some gang members.

iii. On Monday at 19.00, there was an argument between some gang members about whether to sell a high dose of drugs to a customer.

8. Your colleague, who is becoming friends with a KET22 gangster, recently slipped you some details. She said that KET22 is connected to a much bigger gang called TETO. TETO supplies opioids wholesale.

a. Do you have information about the sources from which KET22 obtains narcotics?

b. Has anything about KET22's narcotics operations come to your attention?

i. KET22 is connected to a much bigger gang. 
ii. KET22 is connected to a much bigger gang called TETO.

iii. KET22 is connected to a much bigger gang called TETO that supplies opioids wholesale.

9. KET22 has now added security measures to hide from the police: You discovered this because, after work, you decided to walk home through the woods. You overhead some voices in a secluded area and decided to get a better look. When you got close enough, you recognized that it was some KET22 gangsters. You saw that they were trying to set up some radio communication systems. One of the gangsters was explaining that the communication systems would be used to send alerts about police presence.

a. Have you come across the particular strategies the gang uses to avoid police detection?

b. Have you observed anything about the gang's recent activities?

i. They have adopted security measures to hide from the police.

ii. They have adopted radio communication systems as a security measure to hide from the police.

iii. They have adopted radio communication systems as a security measure to hide from the police; they use the radios to send alerts about police presence.

10. You've noticed that a pickup truck makes deliveries to the KET22 gangsters in the park. The deliveries usually come before the restaurant opens.

a. Do you know the specific ways the gangsters get deliveries in the park?

b. Do you know anything about the gang's drug operations in the park?

i. The gangsters use a pickup truck in the park.

ii. A pickup truck makes deliveries to the gangsters in the park.

iii. A pickup truck makes deliveries to the gangsters in the park before the restaurant opens.

\section{Confidence rating (After each scenario in Study 1 [Designation Experiment])}

- The police-contact asked:

[display question]

- Based on the above question, you selected the option below as what the police-contact wants to know:

[display selection]

- On a scale from $1-5$, how confident are you that your selection is what the police-contact wants to know?

$1=$ not confident at all, $2=$ slightly confident, $3=$ somewhat confident, $4=$ fairly confident, $5=$ completely confident

\section{Confidence measure via bets [optional question]}

- Suppose you were to place a bet on your selection. On a scale from 0 to 100 , what percentage of your compensation (for participating in this research) are you willing to bet that your selection is what the police-contact wants to know. $0 \%=$ none of my compensation, $100 \%=$ all of my compensation 
MENTALLY DESIGNATING INFORMATION ITEMS IN INTERVIEWS

\section{Appendix D}

\section{Control Questions}

The control questions will employ the same scenario outlined below. The scenario will be presented four times, in random order, with four different questions.

Recently, a man came into the restaurant to buy coffee. You suspect he might be one of the KET22 gangsters, but you are unsure. When he made his order, there was no milk at the counter. So, you asked your colleague to get some milk from the fridge in the back. While you were waiting, you got a good look at his face and stature. You can guess that he is about $190 \mathrm{~cm}$ tall. His hair was dark with grey streaks. He had green eyes and a scar on his left jaw. The name on the card he used to pay for his drink was Kari Jupo.

Q1. From the options below, select the name on the card the man used to pay for his drink.

- Minea Blankson

- Johnny Biles

- Kari Jupo

- Renave Olsson

Q2. From the options below, select the correct description of the man's hair.

- Blonde with brown streaks

- Blonde with grey streaks

- Dark with grey streaks

- Dark with yellow streaks

Q3. From the options below, select what the man ordered.

- Sandwich

- Coffee

- Beer

- Salad

Q4. From the options below, select the correct description of the man's height

- $190 \mathrm{~cm}$

- $200 \mathrm{~cm}$

- $164 \mathrm{~cm}$

- $175 \mathrm{~cm}$ 